\title{
Sabotage in team contests
}

\author{
Serhat Doğan ${ }^{1} \cdot$ Kerim Keskin ${ }^{2} \cdot$ Çağrı Sağlam ${ }^{1}$
}

Received: 4 September 2018 / Accepted: 23 January 2019 / Published online: 1 February 2019 (c) Springer Science+Business Media, LLC, part of Springer Nature 2019

\begin{abstract}
In the contest literature, sabotage is defined as a deliberate and costly activity that damages the opponent's likelihood of winning the contest. Most of the existing results suggest that, anticipating a possible sabotage, contestants would be discouraged from exerting high efforts. In this paper we investigate the act of sabotage in a team contest wherein team members exert costly efforts as a contribution to their team's aggregate effort, which in turn determines the contest's outcome. For the baseline model with no sabotage, there exists a corner equilibrium implying a free-rider problem in each team. As for the model with sabotage, our characterization of Nash equilibrium reveals two important results: (i) a unique interior equilibrium exists so that the free-rider problem no longer is a concern and (ii) the discouragement effect of sabotage vanishes for some players. On top of those conclusions, we investigate the team owner's problems of prize allocation and team formation with the objective being to maximize his team's winning probability.
\end{abstract}

Keywords Team contests $\cdot$ Sabotage $\cdot$ Tullock contests $\cdot$ Free riding $\cdot$ Discouragement effect $\cdot$ Encouragement effect

JEL Classification C72 $\cdot$ D74

\section{Introduction}

Contests are strategic interactions in which participants expend costly resources (e.g., effort, time, money) aiming to win a valuable prize. Perhaps what is most important, all of the resources invested are lost independent of who wins the contest. Many real-life examples can be provided, including sports, war, politics, R\&D competition, and advertising. In all of those examples, contestants exert productive efforts in order to increase their chances of winning; and in some of them, contestants also are able to take some actions in order to reduce their opponents' winning probabilities, thereby increasing their own winning probabilities indirectly. The latter action is labeled "sabotage" in the literature.

Kerim Keskin

kerim.keskin@khas.edu.tr

1 Department of Economics, Bilkent University, 06800 Ankara, Turkey

2 Department of Economics, Kadir Has University, 34083 Istanbul, Turkey 
Some actual examples of sabotage are aggressive play or attempting to provoke illegal responses from competitors in sports, destruction of the rival's weaponry or resources in warfare, negative campaigning in politics, and so on. ${ }^{1}$ In addition to such observations, many scholars have reported sabotage in laboratory experiments (see Harbring and Irlenbusch 2005, 2011; Harbring et al. 2007; Vandegrift and Yavas 2010, among others) and field studies (see Balafoutas et al. 2012; Deutscher et al. 2013; Brown and Chowdhury 2017, among others). For example, among such studies, Harbring et al. (2007) investigated behavior in experimental corporate contests with heterogeneous players; whereas Vandegrift and Yavas (2010) examined a similar framework preceded by a real-effort task that endogenized heterogeneity in the participants' ability levels. As for the field studies, Balafoutas et al. (2012) and Deutscher et al. (2013) both reported that sabotage is more likely to be adopted as a strategy by relatively less qualified participants and to be targeted at more qualified ones, analyzing data from the Judo World Championships and German Bundesliga, respectively.

To the best of our knowledge, in the literature on contests/tournaments, Dye (1984) is the first to mention the possibility of sabotage. Lazear (1989) presented a theoretical model of sabotage in contests and showed that a larger prize spread between the winner and the loser(s) would lead to an increase in sabotage activity. ${ }^{2}$ Later, Konrad (2000) studied the effect of sabotage on equilibrium behavior in an $n$-player lobbying contest. He showed that sabotage may raise or lower the total lobbying effort exerted in equilibrium and the total amount of sabotage declines in the number of players. Afterwards, Chen (2003) analyzed sabotage in promotion tournaments where relative performances are important and indicated that the highest caliber players might not have the best chances of being promoted. Münster (2007) studied a case of directed sabotage in selection tournaments and showed that contestants who exert more productive efforts are sabotaged more heavily in equilibrium. He argued further that the possibility of sabotage may even deter the productive players from entering the tournament in the first place. In a related study, Amegashie (2012) analyzed subgame perfect Nash equilibrium of a two-stage contest in which players choose destructive efforts (i.e., sabotage) in stage 1 and productive efforts in stage 2 . He showed that players engage in destructive activities only if the prize for winning is sufficiently large and, beyond that threshold, productive efforts remain constant in the prize level.

Despite the fact that the act of sabotage already has been studied in the contest literature for over 30 years, the analysis of sabotage in team contests remains an understudied topic. To our knowledge, only one paper studies sabotage in a team contest. Gürtler (2008) assumed that each contestant, as a member of one of the two teams, chooses a productive effort in order to increase his team's performance, but also is able to sabotage the members of the opposing team. As a main result, it is shown that a team directs all of its sabotage activities at the least skilled member of the opposing team. That result is rather interesting, since it is in stark contrast to findings that the most skilled players are most likely sabotaged in individual contests (see Chen 2003; Münster 2007).

We investigate sabotage in a one-shot contest between two teams having two members each. The team members differ in their effectiveness parameters and prizes for winning. We can provide several interpretations of that model: (i) Consider two football teams playing

\footnotetext{
1 For more real-life examples, see a recent survey by Chowdhury and Gürtler (2015).

2 A result that is verified observationally by a number of studies (e.g., Garicano and Palacios-Huerta 2014; Harbring and Irlenbusch 2005, 2011; Vandegrift and Yavas 2010).
} 
a game. Each football team fields attackers and defenders. ${ }^{3}$ (ii) Consider two countries at war. Each country has attack forces and defensive forces. (iii) Consider two political parties competing over two regions in an election. The party's representatives in different constituencies can be interpreted as different team members. In the current paper, for the sake of expositional simplicity, we stick to the football game interpretation, keeping the alternative interpretations in mind. Now, in a football team, each team member chooses a productive effort that contributes to his team's aggregate effort, which in turn determines the contest's outcome. Additionally, each team member is able to sabotage a particular member of the opposing team, which we call directionally restricted sabotage. ${ }^{4}$ We then characterize and compare the sets of Nash equilibria in models with no sabotage and with directionally restricted sabotage.

One of the most common results in the literature on sabotage in contests is the discouragement effect (see Sect. 4 of Chowdhury and Gürtler 2015). Along that line, Chen (2003) and Münster (2007) showed that the most skilled players are sabotaged more heavily; Gürtler and Münster (2010) found that players who exerted high effort in the first stage of a two-stage contest are sabotaged more than those who exerted low effort. Those findings imply that if the possibility of being sabotaged is open, incentives are weaker to exert high productive efforts or even to participate in the contest at all. On top of those effects, the above-mentioned findings of Amegashie (2012) imply that sabotage fully crowds out any additional productive effort that would have been supplied by players in the absence of sabotage. In stark contrast to those existing results, in the interior equilibrium of our model, we observe the opposite effect for one of the team members. More precisely, compared to the equilibrium efforts exerted in the model with no sabotage, a team member exists who exerts more productive effort once a sabotage option becomes available. That result, which can be labeled as the encouragement effect, appears to be related to the collective-effort nature of our model.

Another interesting issue is that in the baseline model with no sabotage, we detect a freerider problem. Depending on the values of effectiveness parameters and prizes for winning, either the attackers or defenders supply no productive effort in equilibrium. In fact, similar findings were reported previously by Nitzan (1991), Baik (1993, 2008), and Baik et al. (2001). ${ }^{5}$ Such a result is related to seminal work by Holmström (1982), highlighting freerider problems in a team-production setting (i.e., a group of individuals organized so that the team's output exceeds that of any one team member). The literature emerged from Holmström (1982)'s paper focuses on optimal contract design for solving such free-rider problems and capturing the team's productivity advantages (see McAfee and McMillan 1991; Itoh 1991; Vander Veen 1995; Gershkov et al. 2009, among others). Interestingly, without referring to an optimal contract, in this paper we show that the option to sabotage works as a natural solution to free riding, since the free riding team member starts contributing to his team's aggregate effort once the possibility of sabotage from the opposing team members exists. On

\footnotetext{
3 Treating a group of attackers/defenders as a single decision-maker, we label them as a team member. The same is true for the alternative interpretations that follow.

${ }^{4}$ Sabotage activity is said to be directed if a player is facing multiple opponents and is able to choose the victim of his sabotage. Here we restrict the possible directions for sabotage, arguing that the attackers/defenders in a football team are facing the defenders/attackers in the opposing team. For the interested reader, we analyze the case of directed sabotage in Appendix 2 and characterize the conditions under which the model reduces to our original model with directionally restricted sabotage.

5 Indeed, if one considers our baseline model in the context of public good provision, such results date back to Olson (1965).
} 
a related note, one can argue that not many real-life observations from sports, wars, or elections can be found in which some team members exert no productive effort. Along that line, anecdotal evidence suggests that the introduction of sabotage into the baseline model leads to more accurate predictions regarding such strategic interactions in real-life.

Finally, we are interested in the team owner's problems of optimal design. First, consider the following scenario: If a team wins the contest, the team owner receives a prize; a certain fraction of that prize will be distributed as a premium to the team members. The team owner's problem is to allocate such prize shares optimally in order to maximize his team's winning probability. Second, consider the following scenario: A team owner manages a budget to be spent on attackers and defenders who differ in their effectiveness parameters. Given that hiring more effective players is more costly, the team owner's problem is to form the team optimally in order to maximize his team's winning probability. Here we characterize the team owner's optimal strategies in those two situations.

The rest of the paper is organized as follows: In Sect. 2, we formulate the models with no sabotage and with directionally restricted sabotage. We then characterize and compare their sets of Nash equilibria. In Sect. 3, we investigate the team owner's problems of optimal design by letting the team owner (i) allocate a given prize among team members and (ii) spend a given budget for hiring players with different effectiveness parameters. Section 4 concludes.

\section{The model}

As mentioned earlier, for the sake of expositional simplicity, we build on a football game interpretation in this paper. The alternative interpretations are referred to whenever necessary.

\subsection{A team contest}

Consider two football teams playing a game: team 1 and team 2. Each team consists of two groups: attackers $(a)$ and defenders $(d)$. As we treat each group as a single decision-maker, each group is labeled as a team member. In this football game, each team member decides how much productive effort to exert. Their efforts contribute to the aggregate efforts of their teams, which in turn determine the contest's outcome. If team $i \in\{1,2\}$ wins the contest, then player $j \in\{a, d\}$ in team $i$ gets a prize of $V_{i}^{j}>0$, whereas the members of the losing team do not get any payoff.

Other than their prizes for winning, the team members also differ in the effectiveness of their productive efforts to their team's aggregate effort function. For every team $i \in\{1,2\}$, the aggregate effort is given by

$$
\mathcal{E}_{i}=\gamma_{i}^{a} e_{i}^{a}+\gamma_{i}^{d} e_{i}^{d}
$$

where $e_{i}^{j} \in[0, \infty)$ is the productive effort exerted by player $j \in\{a, d\}$ and $\gamma_{i}^{j}>0$ is the effectiveness parameter for player $j \in\{a, d\}$. The winner is determined by the following Tullock-type contest success function:

$$
P_{i}\left(\mathcal{E}_{1}, \mathcal{E}_{2}\right)=\frac{\mathcal{E}_{i}}{\mathcal{E}_{1}+\mathcal{E}_{2}} .
$$

Finally, for any player $j \in\{a, d\}$ in any team $i \in\{1,2\}$, we consider the same linear costof-effort function: 


$$
C_{i}^{j}\left(e_{i}^{j}\right)=e_{i}^{j}
$$

To sum up, in the specified contest, each player $j \in\{a, d\}$ in each team $i \in\{1,2\}$ maximizes

$$
U_{i}^{j}\left(e_{i}^{j}, \cdot\right)=P_{i}\left(\mathcal{E}_{1}, \mathcal{E}_{2}\right) V_{i}^{j}-C_{i}^{j}\left(e_{i}^{j}\right)=\frac{\mathcal{E}_{i}}{\mathcal{E}_{1}+\mathcal{E}_{2}} V_{i}^{j}-e_{i}^{j} .
$$

Below we analyze the Nash equilibrium for this baseline model.

Proposition 1 In the above-described team contest, assume that for players $j, j^{\prime} \in\{a, d\}$ in team 1 and $k, k^{\prime} \in\{a, d\}$ in team 2 :

$$
\gamma_{1}^{j} V_{1}^{j}>\gamma_{1}^{j^{\prime}} V_{1}^{j^{\prime}} \quad \text { and } \quad \gamma_{2}^{k} V_{2}^{k}>\gamma_{2}^{k^{\prime}} V_{2}^{k^{\prime}}
$$

Then, only a corner equilibrium exists in which

$$
\begin{aligned}
& e_{1}^{j}=\frac{\gamma_{1}^{j} \gamma_{2}^{k}\left(V_{1}^{j}\right)^{2} V_{2}^{k}}{\left(\gamma_{1}^{j} V_{1}^{j}+\gamma_{2}^{k} V_{2}^{k}\right)^{2}}, \quad e_{1}^{j^{\prime}}=0, \\
& e_{2}^{k}=\frac{\gamma_{1}^{j} \gamma_{2}^{k} V_{1}^{j}\left(V_{2}^{k}\right)^{2}}{\left(\gamma_{1}^{j} V_{1}^{j}+\gamma_{2}^{k} V_{2}^{k}\right)^{2}}, \quad e_{2}^{k^{\prime}}=0 .
\end{aligned}
$$

That result leads to the following equilibrium aggregate efforts:

$$
\mathcal{E}_{1}^{*}=\frac{\left(\gamma_{1}^{j}\right)^{2} \gamma_{2}^{k}\left(V_{1}^{j}\right)^{2} V_{2}^{k}}{\left(\gamma_{1}^{j} V_{1}^{j}+\gamma_{2}^{k} V_{2}^{k}\right)^{2}} \quad \text { and } \quad \mathcal{E}_{2}^{*}=\frac{\gamma_{1}^{j}\left(\gamma_{2}^{k}\right)^{2} V_{1}^{j}\left(V_{2}^{k}\right)^{2}}{\left(\gamma_{1}^{j} V_{1}^{j}+\gamma_{2}^{k} V_{2}^{k}\right)^{2}}
$$

Furthermore, if $\gamma_{i}^{a} V_{i}^{a}=\gamma_{i}^{d} V_{i}^{d}$ for some team $i \in\{1,2\}$, then there exist multiple equilibria such that both members of team i exert non-negative productive efforts reaching an aggregate effort of $\mathcal{E}_{i}^{*}$.

Proof The equilibrium of this team contest is already analyzed in the literature (see Nitzan 1991; Baik 1993). In order to make the current paper self-contained, we provide an equilibrium analysis in Appendix 1.

Let $\gamma_{i}^{j} V_{i}^{j}$ denote a measure for motivation of player $j \in\{a, d\}$ in team $i \in\{1,2\}$. The idea is that an increase in $\gamma_{i}^{j}$ or $V_{i}^{j}$ would increase player $j$ 's expected utility, which motivates him to contribute more. Notice that in the statement of Proposition 1 , the players $j$ in team 1 and $k$ in team 2 are assumed to be relatively more motivated in their teams. And apparently, the equilibrium aggregate efforts only depend on the effectiveness parameters and the winning prizes of these more motivated players. In particular, $\mathcal{E}_{1}^{*}$ is increasing in $\gamma_{1}^{j}$ 
Fig. 1 A graphical illustration of the model

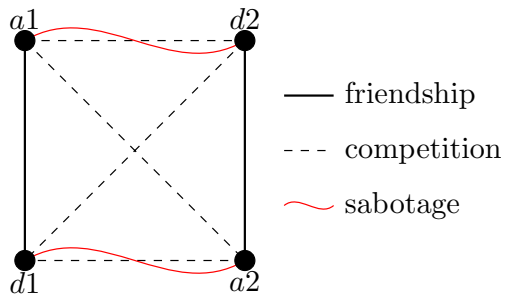

and $V_{1}^{j}$, but decreasing in $\gamma_{2}^{k}$ and $V_{2}^{k}$. The symmetric is true for $\mathcal{E}_{2}^{*}$, and the respective interpretations are quite straightforward.

We complete this section by emphasizing the following remark.

Remark 1 If $\gamma_{i}^{a} V_{i}^{a} \neq \gamma_{i}^{d} V_{i}^{d}$ for some team $i \in\{1,2\}$, then there exists a free riding member of team $i$ exerting no productive effort in the team contest.

This observation becomes particularly important when the option to sabotage is introduced in the following section.

\subsection{Introducing sabotage}

In this section, we introduce an additional choice variable for the team members: sabotage. Any act that reduces the effectiveness of the opposing team members can be classified as a sabotage activity. For instance, given the football game interpretation, some of the possible sabotage activities would be playing more aggressively or attempting to provoke illegal responses from the opposing team members. With such actions one may inflict injuries or may cause the opponent players to receive yellow/red cards. Those would undermine the effectiveness of the opposing team members, thereby indirectly creating an advantage for one's team. ${ }^{6}$

Here we consider a situation where each member of team 1 can sabotage only a particular member of team 2, and vice versa. The intuition is as follows: In a football game, a team's attackers are faced with the opposing team's defenders; if the opposing team's defenders exert some sabotage effort, then the attackers should be the ones suffering from such acts. That is what we call directionally restricted sabotage. ${ }^{7}$

We acknowledge that various ways of introducing sabotage into the baseline model are possible. Here we assume that a sabotage act directly reduces the effectiveness of the victim, which implies a direct reduction in the opposing team's winning probability. For the sake of concreteness, we assume that if player $j \in\{a, d\}$ in team $i \in\{1,2\}$ is sabotaged in the amount of $s \in[0, \infty)$, then player $j$ 's effectiveness reduces to $\gamma_{i}^{j} /(1+s)$. We argue that

\footnotetext{
${ }^{6}$ Following the war interpretation, the destruction of a rival's weaponry or resources can be labeled as a sabotage act. Or, following the election interpretation, a possible sabotage activity would be negative campaigning.

7 From another perspective, we study a team contest played on a small network with (i) four nodes representing the team members and (ii) three types of links representing their interactions (see Fig. 1). In particular, $a 1$ has a friendship link with $d 1$; $a 2$ has a friendship link with $d 2 ; a 1$ and $d 1$ have competition links with both $a 2$ and $d 2$; $a 1$ has a sabotage link with $d 2$; and $d 1$ has a sabotage link with $a 2$.
} 
such an assumption is consistent with our model's interpretations, since it captures the idea that a player cannot possibly reduce the effectiveness of an opposing team member to zero, no matter how much sabotage effort he chooses to exert. Accordingly, the aggregate effort functions become

$$
E_{1}=\frac{\gamma_{1}^{a}}{1+s_{2}^{d}} e_{1}^{a}+\frac{\gamma_{1}^{d}}{1+s_{2}^{a}} e_{1}^{d}
$$

and

$$
E_{2}=\frac{\gamma_{2}^{a}}{1+s_{1}^{d}} e_{2}^{a}+\frac{\gamma_{2}^{d}}{1+s_{1}^{a}} e_{2}^{d}
$$

where $s_{i}^{j} \in[0, \infty)$ denotes the sabotage effort exerted by player $j \in\{a, d\}$ in team $i \in\{1,2\}$ directed at the respective member of the opposing team.

The Tullock-type contest success function is preserved in terms of the aggregate efforts. Finally, the cost-of-effort function of player $j \in\{a, d\}$ in team $i \in\{1,2\}$ is updated to

$$
C_{i}^{j}\left(e_{i}^{j}, s_{i}^{j}\right)=e_{i}^{j}+\mu_{i}^{j} s_{i}^{j}
$$

where $\mu_{i}^{j}>0$ denotes the cost of exerting one unit of sabotage effort.

In the new contest, each player $j \in\{a, d\}$ in each team $i \in\{1,2\}$ maximizes

$$
U_{i}^{j}\left(\left(e_{i}^{j}, s_{i}^{j}\right), \cdot\right)=P_{i}\left(E_{1}, E_{2}\right) V_{i}^{j}-C_{i}^{j}\left(e_{i}^{j}, s_{i}^{j}\right)=\frac{E_{i}}{E_{1}+E_{2}} V_{i}^{j}-e_{i}^{j}-\mu_{i}^{j} s_{i}^{j} .
$$

Below we characterize the unique interior Nash equilibrium of this model.

Proposition 2 In the model with directionally restricted sabotage, the aggregate efforts in the unique interior equilibrium are given by

$$
E_{1}^{*}=\gamma_{1}^{d} \mu_{2}^{a} \frac{V_{1}^{d}}{V_{2}^{a}}+\gamma_{1}^{a} \mu_{2}^{d} \frac{V_{1}^{a}}{V_{2}^{d}} \quad \text { and } \quad E_{2}^{*}=\gamma_{2}^{d} \mu_{1}^{a} \frac{V_{2}^{d}}{V_{1}^{a}}+\gamma_{2}^{a} \mu_{1}^{d} \frac{V_{2}^{a}}{V_{1}^{d}}
$$

Furthermore, the respective productive and sabotage efforts are

$$
\begin{aligned}
& s_{1}^{d *}=\frac{\gamma_{2}^{a} E_{1}^{*} V_{2}^{a}}{\left(E_{1}^{*}+E_{2}^{*}\right)^{2}}-1, \quad s_{1}^{a *}=\frac{\gamma_{2}^{d} E_{1}^{*} V_{2}^{d}}{\left(E_{1}^{*}+E_{2}^{*}\right)^{2}}-1, \\
& s_{2}^{d *}=\frac{\gamma_{1}^{a} E_{2}^{*} V_{1}^{a}}{\left(E_{1}^{*}+E_{2}^{*}\right)^{2}}-1, \quad s_{2}^{a *}=\frac{\gamma_{1}^{d} E_{2}^{*} V_{1}^{d}}{\left(E_{1}^{*}+E_{2}^{*}\right)^{2}}-1, \\
& e_{1}^{d *}=\frac{\mu_{2}^{a} \gamma_{1}^{d} E_{2}^{*}\left(V_{1}^{d}\right)^{2}}{V_{2}^{a}\left(E_{1}^{*}+E_{2}^{*}\right)^{2}}, \quad e_{1}^{a *}=\frac{\mu_{2}^{d} \gamma_{1}^{a} E_{2}^{*}\left(V_{1}^{a}\right)^{2}}{V_{2}^{d}\left(E_{1}^{*}+E_{2}^{*}\right)^{2}}, \\
& e_{2}^{d *}=\frac{\mu_{1}^{a} \gamma_{2}^{d} E_{1}^{*}\left(V_{2}^{d}\right)^{2}}{V_{1}^{a}\left(E_{1}^{*}+E_{2}^{*}\right)^{2}}, \quad e_{2}^{a *}=\frac{\mu_{1}^{d} \gamma_{2}^{a} E_{1}^{*}\left(V_{2}^{a}\right)^{2}}{V_{1}^{d}\left(E_{1}^{*}+E_{2}^{*}\right)^{2}} .
\end{aligned}
$$


Such an interior equilibrium exists if and only if

$$
\frac{\gamma_{i}^{j} E_{-i}^{*} V_{i}^{j}}{\left(E_{1}^{*}+E_{2}^{*}\right)^{2}}>1
$$

for every player $j \in\{a, d\}$ in $i \in\{1,2\}$; and those inequalities are satisfied as long as all of the winning prizes are sufficiently large.

Proof See Appendix 1.

For the comparative statics results, we focus on team 1 . The equilibrium aggregate effort $E_{1}^{*}$ increases in the effectiveness parameters and the winning prizes for both members of team 1, as well as in the marginal costs of sabotage for both members of team 2 . Furthermore, $E_{1}^{*}$ declines when the winning prize for either of the members of team 2 increases.

The comparative statics for the equilibrium values of productive and sabotage efforts are not straightforward. Accordingly, we concentrate on the ratio of productive efforts in the equilibrium. For instance, considering the ratio

$$
\frac{e_{1}^{d *}}{e_{1}^{a *}}=\frac{\mu_{2}^{a} \gamma_{1}^{d} V_{2}^{d}\left(V_{1}^{d}\right)^{2}}{\mu_{2}^{d} \gamma_{1}^{a} V_{2}^{a}\left(V_{1}^{a}\right)^{2}},
$$

we see that the relative productive effort of player $d$ in team 1 increases following an increase in the effectiveness parameter for that player, in the marginal cost of sabotage for the respective saboteur, or in the winning prize for the defenders in either of the teams. The converse would be true for the parameters in the denominator. As for the sabotage activity, considering the ratio

$$
\frac{1+s_{1}^{d *}}{1+s_{1}^{a *}}=\frac{\gamma_{2}^{a} V_{2}^{a}}{\gamma_{2}^{d} V_{2}^{d}},
$$

we see that the relative sabotage effort of player $d$ in team 1 increases with an increase either in the effectiveness parameter or in the winning prize for the respective victim: player $a$ in team 2 . The converse would be true for the parameters in the denominator.

Our model yields an interesting insight regarding free riding. As highlighted in Remark 1, our result in the baseline model is related to the findings in the optimal contract literature following Holmström (1982). This literature focuses on free-rider problems among team members and studies optimal contract design to resolve such problems (see McAfee and McMillan 1991; Itoh 1991; Vander Veen 1995; Gershkov et al. 2009, among others). By contrast, in the current paper, we show that an optimal contract analysis may not be necessary in the sense that none of the team members free rides once they have an option to sabotage their opponents.

Remark 2 Although a free-rider problem materializes in the baseline model with no sabotage, our model with directionally restricted sabotage generates a unique interior 
equilibrium for which none of the team members free rides. That result indicates the possibility of sabotage turning out to be a natural solution to free riding. ${ }^{8}$

The intuition is related to the fact that sabotage reduces the victim's marginal productive effort in the contest success function. That is, if a player is sabotaged more heavily, then his effort would have a smaller impact on his team's probability of winning, and thus he would have less incentive to exert productive effort. At this point, recall that $\gamma_{i}^{j} V_{i}^{j}$ denotes a measure of motivation for player $j \in\{a, d\}$ in team $i \in\{1,2\}$. Then, our equilibrium analysis for the baseline model suggests that the relatively less motivated team member free rides if sabotage is not an option. When sabotage is available, the new motivation of player $d$ in team 1 becomes

$$
\frac{\gamma_{1}^{d}}{1+s_{2}^{a *}} V_{1}^{d}=\frac{\left(E_{1}^{*}+E_{2}^{*}\right)^{2}}{E_{2}^{*}}
$$

in the unique interior equilibrium. The new motivation of player $a$ in team 1 , after being sabotaged, turns out to be the same. Since players $a$ and $d$ are now equally motivated, both are willing to exert positive productive efforts in the equilibrium. It is worth noting that such an outcome does not lead to a multiplicity of equilibria, since the productive efforts should be chosen in such a way that they are consistent with the equilibrium sabotage efforts.

From an efficiency perspective, the foregoing result deserves further discussion. Exerting more effort in a contest with an exogenously given winning prizes arguably is inefficient, since exerting effort is costly without returning any added value, which implies that solving the free-rider problem would be undesirable. Yet, it may be desirable in certain situations wherein third parties (e.g., spectators in sport contests, consumers in a competitive market) benefit from greater contest efforts. Those issues aside, one should keep in mind that, in this paper, the act of sabotage is not proposed as a rule or mechanism for solving the free-rider problem, but rather it turns out to be a natural solution after being introduced as a realistic extension of the baseline model with no sabotage. That consideration highlights that a designer guided by efficiency concerns should be extra careful studying such team contests and should avoid relying on an oversimplified model, ${ }^{9}$ which might be very misleading.

Another important issue is that our findings contradict a common result in the literature on sabotage in contests/tournaments. More precisely, it is argued in the literature that the prospect of being sabotaged creates a discouragement effect in the sense that contestants exert less productive effort relative to the case of no sabotage. By contrast, we show here that the introduction of sabotage does not discourage some players, but it even encourages them. In our baseline model with no sabotage, the more motivated team members exert

\footnotetext{
${ }^{8}$ There is another well-known solution to complete free riding in team contests, even when sabotage is not available. If players have strictly convex cost functions, then it is possible to construct an equilibrium in which both team members exert positive productive efforts (see Esteban and Ray 2001). Accordingly, it can be argued that the introduction of sabotage plays a role analogous to that played by a strictly convex cost function.

9 An oversimplified model refers to a model that disregards the possibility of sabotage although the reallife scenario to be explained includes a sabotage act. Apparently, such an oversimplified model might make significantly different predictions.
} 
positive productive efforts, while the other players choose to free ride. When the possibility of sabotage is introduced, however, the free riding team members are encouraged, as they start contributing to their teams' aggregate efforts. We relate that result back to the collective nature of our contest success function.

Remark 3 For the free riding team members in the baseline model, the possibility of sabotage creates an encouragement effect.

It is worth mentioning that here we concentrate on the productive effort exerted by a specific team member. If we instead analyze total productive effort for team $i \in\{1,2\}$, which can be written as $e_{i}^{a *}+e_{i}^{d *}$, we can say that the possibility of sabotage has a mixed effect. In particular, if the marginal costs of sabotage are sufficiently low, then players have weaker incentives to exert productive efforts as they anticipate high levels of sabotage, which implies lower total productive effort compared to that in the baseline model. That is, although the free riding team member in the baseline model is encouraged, the active team member might be strongly discouraged such that a discouragement effect prevails overall. Conversely, if the marginal costs of sabotage are sufficiently high, then players are inclined to exert more productive efforts anticipating low levels of sabotage. In such a case, an encouragement effect is observed also in total productive efforts.

As mentioned earlier in Footnote 8, if players exhibit strictly convex cost functions, then the free-rider problem disappears in the baseline model with no sabotage. Since it is the free riding team member who is encouraged once a sabotage option becomes available, the following question arises: Would an encouraged player still materialize in the model with sabotage if the cost functions are strictly convex ${ }^{10}$ Since the equilibrium analysis of such a model turns out to be rather intractable, we answer that question by referring to a numerical example. Given the cost-of-effort functions $C_{i}^{j}(e)=e^{2}$ and $C_{i}^{j}(e, s)=e^{2}+\mu_{i}^{j} s^{2}$ for every player $j \in\{a, d\}$ in team $i \in\{1,2\}$ for the respective models, consider the following values of the effectiveness parameters: $\gamma_{1}^{a}=\gamma_{2}^{d}=1$ and $\gamma_{1}^{d}=\gamma_{2}^{a}=2$. Further assume that $\mu_{i}^{j}=1$ and $V_{i}^{j}=10$ for every player $j \in\{a, d\}$ in team $i \in\{1,2\}$. In the baseline model with no sabotage, the equilibrium strategies are

$$
e_{1}^{a}=e_{2}^{d}=0.5, \quad e_{1}^{d}=e_{2}^{a}=1 .
$$

In the model with sabotage, the equilibrium strategies become

$$
\begin{array}{ll}
e_{1}^{a}=e_{2}^{d}=0.588, & e_{1}^{d}=e_{2}^{a}=0.951 \\
s_{1}^{a}=s_{2}^{d}=0.272, & s_{1}^{d}=s_{2}^{a}=0.574 .
\end{array}
$$

Observing that (i) each team has an encouraged player who exerts more productive effort and (ii) each team increases its total productive effort, it is verified numerically that the encouragement effect of sabotage is not necessarily explained by the linear cost functions considered.

Finally, in this paper we study a particular team contest with directionally restricted sabotage, which is played on a network with given sabotage links. We also could consider a case in which each team member can sabotage any member of the opposing team. In order

$\overline{10}$ We thank an anonymous reviewer for bringing this point to our attention. 
to provide some insights into that modification for the interested reader, such an analysis is given in Appendix 2.

\section{Team owner's problems}

In this section we study two optimal design problems for the team owner. Throughout this section, we assume that the most undesirable outcome for a team owner is to have a freerider in his team. It is accordingly assumed that a team owner restricts his attention to cases in which an interior equilibrium exists.

\subsection{Prize allocation}

Assume that the owner of team $i$ decides on how to distribute a total prize of $V_{i}$ among team members $a$ and $d$ in case of winning. Accordingly, the respective constraints can be written as $V_{1}^{a}+V_{1}^{d}=V_{1}$ and $V_{2}^{a}+V_{2}^{d}=V_{2}$. Obviously, given a strategy for the opposing team's owner, the team owner's objective is to maximize his team's winning probability. More precisely, the owner of team 1 maximizes

$$
P_{1}\left(E_{1}, E_{2}\right)=\frac{E_{1}}{E_{1}+E_{2}}=\frac{\gamma_{1}^{d} \mu_{2}^{a} \frac{V_{1}^{d}}{V_{2}^{a}}+\gamma_{1}^{a} \mu_{2}^{d} \frac{V_{1}^{a}}{V_{2}^{d}}}{\left(\gamma_{1}^{d} \mu_{2}^{a} \frac{V_{1}^{d}}{V_{2}^{a}}+\gamma_{1}^{a} \mu_{2}^{d} \frac{V_{1}^{a}}{V_{2}^{d}}\right)+\left(\gamma_{2}^{d} \mu_{1}^{a} \frac{V_{2}^{d}}{V_{1}^{a}}+\gamma_{2}^{a} \mu_{1}^{d} \frac{V_{2}^{a}}{V_{1}^{d}}\right)} .
$$

The following proposition shows the optimal allocation of prize shares.

Proposition 3 In the model with directionally restricted sabotage, the owner of team 1 should allocate a total prize of $V_{1}$ according to

$$
V_{1}^{d *}=\frac{V_{1}}{2}\left(\frac{\gamma_{1}^{d} \mu_{2}^{a}}{\gamma_{1}^{d} \mu_{2}^{a}+\gamma_{1}^{a} \mu_{2}^{d}}+\frac{\gamma_{2}^{a} \mu_{1}^{d}}{\gamma_{2}^{d} \mu_{1}^{a}+\gamma_{2}^{a} \mu_{1}^{d}}\right)
$$

and

$$
V_{1}^{a *}=\frac{V_{1}}{2}\left(\frac{\gamma_{1}^{a} \mu_{2}^{d}}{\gamma_{1}^{d} \mu_{2}^{a}+\gamma_{1}^{a} \mu_{2}^{d}}+\frac{\gamma_{2}^{d} \mu_{1}^{a}}{\gamma_{2}^{d} \mu_{1}^{a}+\gamma_{2}^{a} \mu_{1}^{d}}\right)
$$

in order to maximize his team's winning probability.

Proof See Appendix 1.

For comparative statics, without loss of generality, we concentrate on $V_{1}^{d *}$. It is easy to see that if the expression in parenthesis is greater than 1 , then $V_{1}^{d *}>V_{1} / 2>V_{1}^{a *}$, i.e., the owner of team 1 prefers to allocate a larger prize share to the defenders. That scenario occurs when $\gamma_{1}^{d}, \gamma_{2}^{a}, \mu_{1}^{d}$, and $\mu_{2}^{a}$ are sufficiently high. The conclusion is quite intuitive, since each of the parameters represents the significance of player $d$ in team $1: \gamma_{1}^{d}$ is the 
effectiveness parameter for that player; $\gamma_{2}^{a}$ is the effectiveness parameter for the opposing team player who has a sabotage link to that player; and $\mu_{1}^{d}$ and $\mu_{2}^{a}$ are the respective costs of sabotage for those two players. Accordingly, if $\gamma_{1}^{d}$ increases, since player $d$ in team 1 becomes more effective, he should be incentivized more; if $\mu_{2}^{a}$ increases, since player $d$ in team 1 is less likely to be sabotaged, it is as if his effectiveness increases, so that he should be incentivized more; and if either $\gamma_{2}^{a}$ or $\mu_{1}^{d}$ increases, then the symmetric effects would be observed for player $a$ in team 2, and being the potential saboteur of that player, player $d$ in team 1 should be incentivized even more so.

Let $\gamma_{1}^{d} \mu_{2}^{a}$ be defined as the weighted effectiveness of player $d$ in team 1 . As $\mu_{2}^{a}$ declines, that player is apt to be sabotaged more, which in turn reduces the player's effectiveness; so that the weighted effectiveness somehow captures the effectiveness of a player depending on his adversary. Now, returning back to $V_{1}^{d *}$, we can reinterpret our result: The owner of team 1 allocates half of the prize proportional to the weighted effectiveness of the members of his team and the other half proportional to the weighted effectiveness of their respective adversaries.

\subsection{Team formation}

Here we model the allocation of the team owner's budget. Consider the situation wherein a team owner must decide how to form his team under a given budget constraint. In particular, we let the team owner choose any effectiveness level for each team member: $\gamma^{a}$ and $\gamma^{d}$. Since $\mu_{i}^{j}$ is not a choice variable for the owner of team $i \in\{1,2\}$, in order for this analysis to be meaningful, we assume that $\mu_{1}^{a}=\mu_{2}^{a}=\mu^{d}$ and $\mu_{1}^{d}=\mu_{2}^{d}=\mu^{a}$. ${ }^{11}$

Under the assumption that the cost of hiring a player with an effectiveness parameter $\gamma$ is $\gamma^{\alpha}$ where $\alpha>1$, the owner of team 1 aims to maximize

$$
P_{1}\left(E_{1}, E_{2}\right)=\frac{E_{1}}{E_{1}+E_{2}}=\frac{\gamma_{1}^{d} \mu^{a} \frac{V_{1}^{d}}{V_{2}^{a}}+\gamma_{1}^{a} \mu^{d} \frac{V_{1}^{a}}{V_{2}^{d}}}{\left(\gamma_{1}^{d} \mu^{a} \frac{V_{1}^{d}}{V_{2}^{a}}+\gamma_{1}^{a} \mu^{d} \frac{V_{1}^{a}}{V_{2}^{d}}\right)+\left(\gamma_{2}^{d} \mu^{a} \frac{V_{2}^{d}}{V_{1}^{a}}+\gamma_{2}^{a} \mu^{d} \frac{V_{2}^{a}}{V_{1}^{d}}\right)}
$$

subject to the budget constraint $\left(\gamma_{1}^{a}\right)^{\alpha}+\left(\gamma_{1}^{d}\right)^{\alpha}=\Gamma_{1}$. We note here that $E_{2}$ is independent of $\gamma_{1}^{a}$ and $\gamma_{1}^{d}$; as a result, the maximization problem corresponds to the maximization of $E_{1}{ }^{12}$

The following proposition shows the optimal effectiveness parameters.

Proposition 4 In the model with directionally restricted sabotage, given the budget constraint $\left(\gamma_{1}^{a}\right)^{\alpha}+\left(\gamma_{1}^{d}\right)^{\alpha}=\Gamma_{1}$, the owner of team 1 should form his team in such a way that

\footnotetext{
11 Suppose that the effectiveness parameters could differ across teams and assume without loss of generality that $\mu_{1}^{d}>\mu_{2}^{d}$. That assumption implies that team 1 cannot hire a defender with a sabotage cost lower than that of the defenders in team 2 . This conclusion surely sounds odd. Here we simply assume that $\mu^{j}$ is a property of a player, but not a team.

12 The setup eliminates the strategic interaction between team owners. Independent of what the owner of team $-i$ does, the owner of team $i$ would always choose the same values of effectiveness parameters for his team's attackers and defenders.
} 


$$
\gamma_{1}^{a *}=\left(\frac{\Gamma_{1}}{1+\left(\frac{\mu^{a} V_{1}^{d} V_{2}^{d}}{\mu^{d} V_{2}^{a} V_{1}^{a}}\right)^{\frac{\alpha}{\alpha-1}}}\right)^{\frac{1}{\alpha}} \text { and } \quad \gamma_{1}^{d *}=\left(\frac{\Gamma_{1}}{1+\left(\frac{\mu^{d} V_{2}^{a} V_{1}^{a}}{\mu^{a} V_{1}^{d} V_{2}^{d}}\right)^{\frac{\alpha}{\alpha-1}}}\right)^{\frac{1}{\alpha}}
$$

in order to maximize his team's winning probability.

Proof See Appendix 1.

We see that the optimal choice of effectiveness parameter for player $d$ in team 1 increases in $\mu^{a}, V_{1}^{d}, V_{2}^{d}$, and $\Gamma_{1}$, whereas it declines in $\mu^{d}, V_{1}^{a}, V_{2}^{a}$, and $\alpha$. Here we omit the interpretations of $\Gamma_{1}$ and $\alpha$, as they seem to be straightforward. The owner of team 1 invests more on the defensive side when $(i) \mu^{a}$ increases, which makes the sabotage by player $a$ in team 2 more costly; (ii) $V_{1}^{d}$ increases, which motivates player $d$ in team 1 to exert more productive effort; and (iii) $V_{2}^{d}$ increases, which deters player $a$ in team 2 from exerting more productive effort, so that player $d$ in team 1 can concentrate further on his productive effort rather than on his sabotage effort. The converse interpretations follow for $\mu^{d}, V_{1}^{a}$, and $V_{2}^{a}$.

Given Proposition 4, we also have

$$
\frac{\gamma_{1}^{d *}}{\gamma_{1}^{a *}}=\left(\frac{\mu^{a} V_{1}^{d} V_{2}^{d}}{\mu^{d} V_{2}^{a} V_{1}^{a}}\right)^{\frac{1}{\alpha-1}}
$$

The foregoing result means that when $\mu^{a} V_{1}^{d} V_{2}^{d}>\mu^{d} V_{2}^{a} V_{1}^{a}$, the owner of team 1 focuses more on the defensive side. That happens when the marginal cost of sabotage is higher for the respective saboteurs and/or when the defenders' prize for winning in either of the teams is higher. On top of those conclusions, the difference between $\gamma_{1}^{d *}$ and $\gamma_{1}^{a *}$ falls as the hiring cost parameter $\alpha$ increases.

In the analysis above, we assume that winning prizes $V_{i}^{j}$ are given exogenously. On the other hand, if we allow endogenous prizes (referring to the analysis in Sect. 3.1), since we would have $V_{1}^{d} V_{2}^{d}=V_{2}^{a} V_{1}^{a}$, the new ratio of $\gamma_{1}^{d *}$ to $\gamma_{1}^{a *}$ would become

$$
\frac{\gamma_{1}^{d *}}{\gamma_{1}^{a *}}=\left(\frac{\mu^{a}}{\mu^{d}}\right)^{\frac{1}{\alpha-1}} \text {. }
$$

That observation leads to the following remark.

Remark 4 In the model with directionally restricted sabotage, given the budget constraint $\left(\gamma_{1}^{a}\right)^{\alpha}+\left(\gamma_{1}^{d}\right)^{\alpha}=\Gamma_{1}$, if team owners choose the allocation of prize shares strategically, then the owner of team 1 should form his team in such a way that

$$
\gamma_{1}^{a *}=\left(\frac{\Gamma_{1}}{1+\left(\frac{\mu^{a}}{\mu^{d}}\right)^{\frac{\alpha}{\alpha-1}}}\right)^{\frac{1}{\alpha}} \text { and } \gamma_{1}^{d *}=\left(\frac{\Gamma_{1}}{1+\left(\frac{\mu^{d}}{\mu^{a}}\right)^{\frac{\alpha}{\alpha-1}}}\right)^{\frac{1}{\alpha}}
$$


in order to maximize his team's winning probability.

It appears that when the winning prizes for both team members are determined endogenously by team owners, the effects of winning prizes on the optimal choices of effectiveness parameters are suppressed. However, the rest of the aforementioned interpretations are preserved.

\section{Conclusion}

In this study, we have contributed to the burgeoning literature on team contests by introducing sabotage as an additional dimension of contestants' strategy spaces. The members of a team choose not only their productive efforts, which contribute to their team's aggregate effort, but also sabotage efforts directed at a particular member of the opposing team. Our analysis unveils two fundamental differences in equilibrium behavior: (i) the discouragement effect of sabotage reported in contests between individuals does not appear for some players in the team contest considered herein; even more interestingly, (ii) the freerider problem inherent in team contests disappears with the added option to sabotage.

The foregoing results highlight the undesirable consequences of ignoring for the sake of simplicity a factor that could be involved in the strategic trade-offs of players. For instance, in this paper we have observed that analyzing strategic interaction between teams (which naturally includes acts of sabotage) in an oversimplified model in which team members are allowed only to choose their productive efforts may create a free-rider problem that in fact does not exist. That conclusion indicates that such an oversimplified model might mislead a designer who is concerned about free riding or who values the intensity of competition between teams, and therefore the designer should not disregard the effect of sabotage on the players' effort choices. Additionally, as sabotage turns out to be a natural solution to the free-rider problem, our model allows us to investigate two different design problems for a team owner: (i) allocation of prize shares among team members and (ii) team formation under a given budget. ${ }^{13}$

Finally, our results also are of interest from an experimental design perspective. Our theoretical predictions will be of practical value to experimental economists who investigate team contests in the lab. Future work may elaborate on this issue.

Acknowledgements We would like to thank the editor and an anonymous reviewer, as well as seminar participants at ADA University, Bilgi University, Bosphorus Workshop on Economic Design, and Koç University Winter Workshop in Economics. The usual disclaimer applies.

\section{Appendix 1}

Proof of Proposition 1 Given an aggregate effort $\mathcal{E}_{2}$ for team 2, the first-order condition with respect to $e_{1}^{a}$ for player $a$ in team 1 is

$$
\frac{\gamma_{1}^{a} \mathcal{E}_{2}}{\left(\mathcal{E}_{1}+\mathcal{E}_{2}\right)^{2}} V_{1}^{a}-1=0 \text {. }
$$

\footnotetext{
13 In the baseline model with no sabotage, the free riding result makes the team owner's problems trivial, since the team owner would concentrate on the reward for the most motivated member of his team.
} 
For player $d$ in team 1, a symmetric first-order condition can be written as

$$
\frac{\gamma_{1}^{d} \mathcal{E}_{2}}{\left(\mathcal{E}_{1}+\mathcal{E}_{2}\right)^{2}} V_{1}^{d}-1=0
$$

Accordingly, it must be that

$$
\gamma_{1}^{a} V_{1}^{a}=\gamma_{1}^{d} V_{1}^{d}
$$

in the equilibrium, which is not necessarily true. That leads to a corner solution such that if the right-hand side exceeds the left-hand side, then only the attackers exert positive productive effort in the equilibrium, and vice versa. Considering a symmetric result for the other team, and under the assumption that $\gamma_{1}^{j} V_{1}^{j}>\gamma_{1}^{j^{\prime}} V_{1}^{j^{\prime}}$ and $\gamma_{2}^{k} V_{2}^{k}>\gamma_{2}^{k^{\prime}} V_{2}^{k^{\prime}}$, the respective equilibrium efforts are

$$
\begin{aligned}
& e_{1}^{j}=\frac{\gamma_{1}^{j} \gamma_{2}^{k}\left(V_{1}^{j}\right)^{2} V_{2}^{k}}{\left(\gamma_{1}^{j} V_{1}^{j}+\gamma_{2}^{k} V_{2}^{k}\right)^{2}}, \quad e_{1}^{j^{\prime}}=0, \\
& e_{2}^{k}=\frac{\gamma_{1}^{j} \gamma_{2}^{k} V_{1}^{j}\left(V_{2}^{k}\right)^{2}}{\left(\gamma_{1}^{j} V_{1}^{j}+\gamma_{2}^{k} V_{2}^{k}\right)^{2}}, \quad e_{2}^{k^{\prime}}=0 .
\end{aligned}
$$

so that

$$
\mathcal{E}_{1}^{*}=\frac{\left(\gamma_{1}^{j}\right)^{2} \gamma_{2}^{k}\left(V_{1}^{j}\right)^{2} V_{2}^{k}}{\left(\gamma_{1}^{j} V_{1}^{j}+\gamma_{2}^{k} V_{2}^{k}\right)^{2}}, \quad \text { and } \quad \mathcal{E}_{2}^{*}=\frac{\gamma_{1}^{j}\left(\gamma_{2}^{k}\right)^{2} V_{1}^{j}\left(V_{2}^{k}\right)^{2}}{\left(\gamma_{1}^{j} V_{1}^{j}+\gamma_{2}^{k} V_{2}^{k}\right)^{2}}
$$

Finally, for the sake of completeness, we must note that if Eq. (1) indeed holds for team $i \in\{1,2\}$, then multiple equilibria exist such that both members of team $i$ exert non-negative productive efforts reaching an aggregate effort of $\mathcal{E}_{i}^{*}$.

Proof of Proposition 2 Consider the maximization problems for players $d$ in team 1 and $a$ in team 2 . The corresponding first-order conditions can be written as

$$
\begin{gathered}
\frac{\partial U_{1}^{d}}{\partial e_{1}^{d}}=\frac{\gamma_{1}^{d}}{1+s_{2}^{a}} \frac{E_{2}}{\left(E_{1}+E_{2}\right)^{2}} V_{1}^{d}-1=0 \\
\frac{\partial U_{1}^{d}}{\partial s_{1}^{d}}=\frac{\gamma_{2}^{a}}{\left(1+s_{1}^{d}\right)^{2}} \frac{e_{2}^{a} E_{1}}{\left(E_{1}+E_{2}\right)^{2}} V_{1}^{d}-\mu_{1}^{d}=0
\end{gathered}
$$




$$
\begin{gathered}
\frac{\partial U_{2}^{a}}{\partial e_{2}^{a}}=\frac{\gamma_{2}^{a}}{1+s_{1}^{d}} \frac{E_{1}}{\left(E_{1}+E_{2}\right)^{2}} V_{2}^{a}-1=0 \\
\frac{\partial U_{2}^{a}}{\partial s_{2}^{a}}=\frac{\gamma_{1}^{d}}{\left(1+s_{2}^{a}\right)^{2}} \frac{e_{1}^{d} E_{2}}{\left(E_{1}+E_{2}\right)^{2}} V_{2}^{a}-\mu_{2}^{a}=0
\end{gathered}
$$

From (3) we get

$$
\frac{\gamma_{1}^{d} E_{2} V_{1}^{d}}{\left(E_{1}+E_{2}\right)^{2}}=1+s_{2}^{a} \text {; }
$$

and from (6) we get

$$
\frac{\gamma_{1}^{d} E_{2} V_{2}^{a}}{\left(E_{1}+E_{2}\right)^{2}}=\mu_{2}^{a} \frac{\left(1+s_{2}^{a}\right)^{2}}{e_{1}^{d}} .
$$

Thus

$$
e_{1}^{d}=\frac{\mu_{2}^{a} \gamma_{1}^{d} E_{2}\left(V_{1}^{d}\right)^{2}}{V_{2}^{a}\left(E_{1}+E_{2}\right)^{2}} .
$$

In a similar manner, we can write all productive and sabotage efforts in terms of each $\gamma_{i}^{j}$, $V_{i}^{j}$, $\mu_{i}^{j}$, and $E_{i}$. The values are

$$
\begin{gathered}
1+s_{1}^{d}=\frac{\gamma_{2}^{a} E_{1} V_{2}^{a}}{\left(E_{1}+E_{2}\right)^{2}}, \quad 1+s_{1}^{a}=\frac{\gamma_{2}^{d} E_{1} V_{2}^{d}}{\left(E_{1}+E_{2}\right)^{2}}, \\
1+s_{2}^{d}=\frac{\gamma_{1}^{a} E_{2} V_{1}^{a}}{\left(E_{1}+E_{2}\right)^{2}}, \quad 1+s_{2}^{a}=\frac{\gamma_{1}^{d} E_{2} V_{1}^{d}}{\left(E_{1}+E_{2}\right)^{2}}, \\
e_{1}^{d}=\frac{\mu_{2}^{a} \gamma_{1}^{d} E_{2}\left(V_{1}^{d}\right)^{2}}{V_{2}^{a}\left(E_{1}+E_{2}\right)^{2}}, \quad e_{1}^{a}=\frac{\mu_{2}^{d} \gamma_{1}^{a} E_{2}\left(V_{1}^{a}\right)^{2}}{V_{2}^{d}\left(E_{1}+E_{2}\right)^{2}}, \\
e_{2}^{d}=\frac{\mu_{1}^{a} \gamma_{2}^{d} E_{1}\left(V_{2}^{d}\right)^{2}}{V_{1}^{a}\left(E_{1}+E_{2}\right)^{2}}, \quad e_{2}^{a}=\frac{\mu_{1}^{d} \gamma_{2}^{a} E_{1}\left(V_{2}^{a}\right)^{2}}{V_{1}^{d}\left(E_{1}+E_{2}\right)^{2}} .
\end{gathered}
$$


Notice that we also have

$$
\frac{e_{2}^{a}}{\left(1+s_{1}^{d}\right)}=\mu_{1}^{d} \frac{V_{2}^{a}}{V_{1}^{d}}
$$

and by symmetry,

$$
\frac{e_{1}^{a}}{\left(1+s_{2}^{d}\right)}=\mu_{2}^{d} \frac{V_{1}^{a}}{V_{2}^{d}} ; \frac{e_{2}^{d}}{\left(1+s_{1}^{a}\right)}=\mu_{1}^{a} \frac{V_{2}^{d}}{V_{1}^{a}} \quad \text { and } \frac{e_{1}^{d}}{\left(1+s_{2}^{a}\right)}=\mu_{2}^{a} \frac{V_{1}^{d}}{V_{2}^{a}}
$$

Thus,

$$
E_{1}^{*}=\gamma_{1}^{d} \frac{e_{1}^{d}}{1+s_{2}^{a}}+\gamma_{1}^{a} \frac{e_{1}^{a}}{1+s_{2}^{d}}=\gamma_{1}^{d} \mu_{2}^{a} \frac{V_{1}^{d}}{V_{2}^{a}}+\gamma_{1}^{a} \mu_{2}^{d} \frac{V_{1}^{a}}{V_{2}^{d}}
$$

and

$$
E_{2}^{*}=\gamma_{2}^{d} \frac{e_{2}^{d}}{1+s_{1}^{a}}+\gamma_{2}^{a} \frac{e_{2}^{a}}{1+s_{1}^{d}}=\gamma_{2}^{d} \mu_{1}^{a} \frac{V_{2}^{d}}{V_{1}^{a}}+\gamma_{2}^{a} \mu_{1}^{d} \frac{V_{2}^{a}}{V_{1}^{d}} .
$$

By replacing those $E_{1}^{*}$ and $E_{2}^{*}$ values into Eqs. (7)-(10), we can write the equilibrium values of all productive and sabotage efforts.

Finally, from Eqs. (7)-(10), we can derive the necessary and sufficient conditions for the existence of an interior equilibrium: Given positive aggregate efforts for both teams, an interior equilibrium exists if and only if for every player $j \in\{a, d\}$ in team $i \in\{1,2\}$, we have $s_{i}^{j}>0$, i.e.,

$$
\frac{\gamma_{i}^{j} E_{-i} V_{i}^{j}}{\left(E_{1}+E_{2}\right)^{2}}>1
$$

And those inequalities are satisfied easily if all of the winning prizes are sufficiently large. ${ }^{14}$ This completes the proof.

Proof of Proposition 3 For given values of $\gamma_{i}^{j}$ and $\mu_{i}^{j}$ for every player $j \in\{a, d\}$ in team $i \in\{1,2\}$, the owner of team 1 aims to maximize

$$
P_{1}\left(E_{1}, E_{2}\right)=\frac{E_{1}}{E_{1}+E_{2}}=\frac{\gamma_{1}^{d} \mu_{2}^{a} \frac{V_{1}^{d}}{V_{2}^{a}}+\gamma_{1}^{a} \mu_{2}^{d} \frac{V_{1}^{a}}{V_{2}^{d}}}{\left(\gamma_{1}^{d} \mu_{2}^{a} \frac{V_{1}^{d}}{V_{2}^{a}}+\gamma_{1}^{a} \mu_{2}^{d} \frac{V_{1}^{a}}{V_{2}^{d}}\right)+\left(\gamma_{2}^{d} \mu_{1}^{a} \frac{V_{2}^{d}}{V_{1}^{a}}+\gamma_{2}^{a} \mu_{1}^{d} \frac{V_{2}^{a}}{V_{1}^{d}}\right)} .
$$

\footnotetext{
${ }^{14}$ Notice that if all winning prizes are multiplied by the same scalar, then the equilibrium values for $E_{1}$ and $E_{2}$ remain unchanged. Accordingly, for any quadruple of winning prizes, a scalar can be found above which the respective winning prizes lead to positive sabotage efforts for all players.
} 
The first-order condition for team 1 yields ${ }^{15}$

$$
\frac{\partial E_{1}}{\partial V_{1}^{d}} E_{2}=\frac{\partial E_{2}}{\partial V_{1}^{d}} E_{1}
$$

Now, considering that $V_{1}^{a}$ and $V_{1}^{d}$ are dependent variables, we have

$$
\left(\gamma_{1}^{d} \mu_{2}^{a} \frac{1}{V_{2}^{a}}-\gamma_{1}^{a} \mu_{2}^{d} \frac{1}{V_{2}^{d}}\right) E_{2}=\left(\gamma_{2}^{d} \mu_{1}^{a} \frac{V_{2}^{d}}{\left(V_{1}^{a}\right)^{2}}-\gamma_{2}^{a} \mu_{1}^{d} \frac{V_{2}^{a}}{\left(V_{1}^{d}\right)^{2}}\right) E_{1}
$$

Similarly, for team 2 we get

$$
\left(\gamma_{2}^{d} \mu_{1}^{a} \frac{1}{V_{1}^{a}}-\gamma_{2}^{a} \mu_{1}^{d} \frac{1}{V_{1}^{d}}\right) E_{1}=\left(\gamma_{1}^{d} \mu_{2}^{a} \frac{V_{1}^{d}}{\left(V_{2}^{a}\right)^{2}}-\gamma_{1}^{a} \mu_{2}^{d} \frac{V_{1}^{a}}{\left(V_{2}^{d}\right)^{2}}\right) E_{2}
$$

After arranging terms, we are left with

$$
\left[\gamma_{1}^{d} \gamma_{2}^{d} \mu_{1}^{a} \mu_{2}^{a}\left(V_{1}^{d} V_{2}^{d}\right)^{2}+\gamma_{1}^{a} \gamma_{2}^{a} \mu_{1}^{d} \mu_{2}^{d}\left(V_{1}^{a} V_{2}^{a}\right)^{2}\right]\left(V_{1}^{d} V_{2}^{d}-V_{1}^{a} V_{2}^{a}\right)=0 .
$$

Since the first term is positive, it must be that $V_{1}^{d} V_{2}^{d}=V_{1}^{a} V_{2}^{a}$. From this equation we find that

$$
\frac{V_{1}^{d}}{V_{2}^{a}}=\frac{V_{1}^{a}}{V_{2}^{d}}=\frac{V_{1}^{d}+V_{1}^{a}}{V_{2}^{d}+V_{2}^{a}}=\frac{V_{1}}{V_{2}}
$$

Then, for the sake of expositional simplicity, we set

$$
\rho_{1}^{d}=\gamma_{1}^{d} \mu_{2}^{a}, \quad \rho_{2}^{d}=\gamma_{2}^{d} \mu_{1}^{a}, \quad \rho_{1}^{a}=\gamma_{1}^{a} \mu_{2}^{d}, \quad \text { and } \rho_{2}^{a}=\gamma_{2}^{a} \mu_{1}^{d} .
$$

Now we can write

$$
E_{1}=\gamma_{1}^{d} \mu_{2}^{a} \frac{V_{1}^{d}}{V_{2}^{a}}+\gamma_{1}^{a} \mu_{2}^{d} \frac{V_{1}^{a}}{V_{2}^{d}}=\frac{V_{1}}{V_{2}}\left(\rho_{1}^{d}+\rho_{1}^{a}\right)
$$

15 Note that

$$
\left(\frac{f(x)}{f(x)+g(x)}\right)^{\prime}=\frac{f^{\prime}(x)(f(x)+g(x))-f(x)\left(f(x)^{\prime}+g(x)^{\prime}\right)}{(f(x)+g(x))^{2}}=\frac{f^{\prime}(x) g(x)-f(x) g^{\prime}(x)}{(f(x)+g(x))^{2}},
$$

so that in order for this derivative to be equal to zero, it must be that

$$
f^{\prime}(x) g(x)=f(x) g^{\prime}(x) .
$$


and

$$
E_{2}=\gamma_{2}^{d} \mu_{1}^{a} \frac{V_{2}^{d}}{V_{1}^{a}}+\gamma_{2}^{a} \mu_{1}^{d} \frac{V_{2}^{a}}{V_{1}^{d}}=\frac{V_{2}}{V_{1}}\left(\rho_{2}^{d}+\rho_{2}^{a}\right)
$$

Following some algebraic operations, the first-order condition for team 1 can be rewritten as

$$
\left(\rho_{1}^{d} \frac{V_{1}}{V_{2} V_{1}^{d}}-\rho_{1}^{a} \frac{V_{1}}{V_{2} V_{1}^{a}}\right) \frac{V_{2}}{V_{1}}\left(\rho_{2}^{d}+\rho_{2}^{a}\right)=\left(\rho_{2}^{d} \frac{V_{2}}{V_{1} V_{1}^{a}}-\rho_{2}^{a} \frac{V_{2}}{V_{1} V_{1}^{d}}\right) \frac{V_{1}}{V_{2}}\left(\rho_{1}^{d}+\rho_{1}^{a}\right)
$$

Canceling out all $V_{1}$ and $V_{2}$, we have

$$
\frac{\rho_{1}^{d}\left(\rho_{2}^{d}+\rho_{2}^{a}\right)+\rho_{2}^{a}\left(\rho_{1}^{d}+\rho_{1}^{a}\right)}{V_{1}^{d}}=\frac{\rho_{2}^{d}\left(\rho_{1}^{d}+\rho_{1}^{a}\right)+\rho_{1}^{a}\left(\rho_{2}^{d}+\rho_{2}^{a}\right)}{V_{1}^{a}} .
$$

From this equality we find

$$
V_{1}^{d}=\frac{V_{1}\left[\rho_{1}^{d}\left(\rho_{2}^{d}+\rho_{2}^{a}\right)+\rho_{2}^{a}\left(\rho_{1}^{d}+\rho_{1}^{a}\right)\right]}{2\left(\rho_{2}^{d}+\rho_{2}^{a}\right)\left(\rho_{1}^{d}+\rho_{1}^{a}\right)}=\frac{V_{1}}{2}\left(\frac{\rho_{1}^{d}}{\rho_{1}^{d}+\rho_{1}^{a}}+\frac{\rho_{2}^{a}}{\rho_{2}^{d}+\rho_{2}^{a}}\right) .
$$

Finally, returning back to the standard notation, we have

$$
V_{1}^{d *}=\frac{V_{1}}{2}\left(\frac{\gamma_{1}^{d} \mu_{2}^{a}}{\gamma_{1}^{d} \mu_{2}^{a}+\gamma_{1}^{a} \mu_{2}^{d}}+\frac{\gamma_{2}^{a} \mu_{1}^{d}}{\gamma_{2}^{d} \mu_{1}^{a}+\gamma_{2}^{a} \mu_{1}^{d}}\right)
$$

The optimal share for the other player is $V_{1}^{a *}=V_{1}-V_{1}^{d *}$.

Proof of Proposition 4 Now that the strategic interaction is absent, the analysis turns out to be much simpler. The first-order condition with respect to $\gamma_{1}^{d}$ is

$$
\mu^{a} \frac{V_{1}^{d}}{V_{2}^{a}}+\mu^{d} \frac{V_{1}^{a}}{V_{2}^{d}} \frac{\partial \gamma_{1}^{a}}{\partial \gamma_{1}^{d}}=0
$$

Moreover, from the derivative of the budget constraint, it follows that

$$
\alpha\left(\gamma_{1}^{a}\right)^{\alpha-1} \frac{\partial \gamma_{1}^{a}}{\partial \gamma_{1}^{d}}+\alpha\left(\gamma_{1}^{d}\right)^{\alpha-1}=0
$$


which implies

$$
\frac{\partial \gamma_{1}^{a}}{\partial \gamma_{1}^{d}}=-\left(\frac{\gamma_{1}^{d}}{\gamma_{1}^{a}}\right)^{\alpha-1}
$$

Using this information in the first-order condition above, we have

$$
\mu^{a} \frac{V_{1}^{d}}{V_{2}^{a}}-\mu^{d} \frac{V_{1}^{a}}{V_{2}^{d}}\left(\frac{\gamma_{1}^{d}}{\gamma_{1}^{a}}\right)^{\alpha-1}=0
$$

so that

$$
\frac{\gamma_{1}^{d}}{\gamma_{1}^{a}}=\left(\frac{\mu^{a} V_{1}^{d} V_{2}^{d}}{\mu^{d} V_{2}^{a} V_{1}^{a}}\right)^{\frac{1}{\alpha-1}}
$$

Then putting this finding into the budget constraint, we have

$$
\gamma_{1}^{a *}=\left(\frac{\Gamma_{1}}{1+\left(\frac{\mu^{a} V_{1}^{d} V_{2}^{d}}{\mu^{d} V_{2}^{a} V_{1}^{a}}\right)^{\frac{\alpha}{\alpha-1}}}\right)^{\frac{1}{\alpha}} \text { and } \gamma_{1}^{d *}=\left(\frac{\Gamma_{1}}{1+\left(\frac{\mu^{d} V_{2}^{a} V_{1}^{a}}{\mu^{a} V_{1}^{d} V_{2}^{d}}\right)^{\frac{\alpha}{\alpha-1}}}\right)^{\frac{1}{\alpha}}
$$

\section{Appendix 2}

\section{The alternative model with restricted sabotage}

In this paper, we have considered directionally restricted sabotage allowing each member of a team to sabotage only a particular member of the opposing team. Here we relax that assumption and analyze the case of directed sabotage: each team member can sabotage any member of the opposing team. Similar to our original model, $e_{i}^{j}$ denotes the productive effort exerted by player $j \in\{a, d\}$ in team $i \in\{1,2\}$. As for the sabotage efforts, we need a new notation including the origin and the destination of sabotage. Let $s_{i}^{j k}$ denote the sabotage made by player $j \in\{a, d\}$ in team $i \in\{1,2\}$ against player $k \in\{a, d\}$ in the opposing team. Also let $\mu_{i}^{j k}$ denote the corresponding marginal cost of such sabotage activity. 
Then, we can write the respective aggregate effort functions as follows:

$$
E_{1}=\frac{\gamma_{1}^{a}}{1+s_{2}^{a a}+s_{2}^{d a}} e_{1}^{a}+\frac{\gamma_{1}^{d}}{1+s_{2}^{a d}+s_{2}^{d d}} e_{1}^{d}
$$

and

$$
E_{2}=\frac{\gamma_{2}^{a}}{1+s_{1}^{a a}+s_{1}^{d a}} e_{2}^{a}+\frac{\gamma_{2}^{d}}{1+s_{1}^{a d}+s_{1}^{d d}} e_{2}^{d} .
$$

Assuming that

$$
C_{i}^{j}\left(e_{i}^{j}, s_{i}^{j a}, s_{i}^{j d}\right)=e_{i}^{j}+\mu_{i}^{j a} s_{i}^{j a}+\mu_{i}^{j d} s_{i}^{j d},
$$

we say that player $j \in\{a, d\}$ in team $i \in\{1,2\}$ maximizes

$$
U_{i}^{j}\left(\left(e_{i}^{j}, s_{i}^{j a}, s_{i}^{j d}\right), \cdot\right)=\frac{E_{i}}{E_{1}+E_{2}} V_{i}^{j}-e_{i}^{j}-\mu_{i}^{j a} s_{i}^{j a}-\mu_{i}^{j d} s_{i}^{j d} .
$$

Utilizing the first-order conditions with respect to $s_{1}^{a a}$ and $s_{1}^{d a}$, we get

$$
\begin{aligned}
& \frac{\partial U_{1}^{a}}{\partial s_{1}^{a a}}=\frac{\gamma_{2}^{a}}{\left(1+s_{1}^{d a}+s_{1}^{a a}\right)^{2}} \frac{e_{2}^{a} E_{1}}{\left(E_{1}+E_{2}\right)^{2}} V_{1}^{a}-\mu_{1}^{a a}=0 \\
& \frac{\partial U_{1}^{d}}{\partial s_{1}^{d a}}=\frac{\gamma_{2}^{a}}{\left(1+s_{1}^{d a}+s_{1}^{a a}\right)^{2}} \frac{e_{2}^{a} E_{1}}{\left(E_{1}+E_{2}\right)^{2}} V_{1}^{d}-\mu_{1}^{d a}=0 .
\end{aligned}
$$

In order for these first-order conditions to be satisfied simultaneously, it must be that

$$
\frac{V_{1}^{a}}{\mu_{1}^{a a}}=\frac{V_{1}^{d}}{\mu_{1}^{d a}}
$$

Otherwise, we must have a corner solution. To put it differently, unless the last equality is satisfied, directionally restricted sabotage would be observed in the equilibrium. Below we elaborate further on that issue.

Note that if any of the derivatives with respect to $s_{1}^{a a}$ and $s_{1}^{d a}$ are positive, then a marginal increment in the corresponding variable would be a possible deviation. Therefore, none can be positive at an equilibrium. The result implies that if one of the first-order conditions is satisfied, then the derivative with respect to the other variable should be negative, which corresponds to a corner solution for that variable. For more concrete arguments, assume without loss of generality that

$$
\frac{V_{1}^{a}}{\mu_{1}^{a a}}<\frac{V_{1}^{d}}{\mu_{1}^{d a}}
$$


If the former first-order condition is satisfied, then the derivative with respect to $s_{1}^{d a}$ would be positive. That cannot happen in equilibrium. Then, it must be that the latter first-order condition is satisfied, meaning that a corner solution exists for $s_{1}^{a a}$, which is $s_{1}^{a a}=0$.

Finally, given our model's interpretation, it is reasonable to assume that $\mu_{1}^{a a}>\mu_{1}^{d a}$. That is because the defenders in team 1 are located closer to the attackers in team 2 than the attackers in team 1 , so that if the attackers in team 2 are to be sabotaged, the defenders in team 1 should have a lower cost than the attackers in team $1 .{ }^{16}$ Accordingly, for a wide range of $V_{1}^{a}, V_{1}^{d}, V_{2}^{a}$, and $V_{2}^{d}$ values, the current model would reduce to our original model with directionally restricted sabotage in the equilibrium.

\section{References}

Amegashie, J. A. (2012). Productive versus destructive efforts in contests. European Journal of Political Economy, 28, 461-468.

Baik, K. H. (1993). Effort levels in contests: The public-good prize case. Economics Letters, 41, 363-367.

Baik, K. H. (2008). Contests with group-specific public-good prizes. Social Choice and Welfare, 30, $103-117$.

Baik, K. H., Kim, I. G., \& Na, S. (2001). Bidding for a group-specific public-good prize. Journal of Public Economics, 82, 415-429.

Balafoutas, L., Lindner, F., \& Sutter, M. (2012). Sabotage in tournaments: Evidence from a natural experiment. Kyklos, 65, 425-441.

Brown, A., \& Chowdhury, S. M. (2017). The hidden perils of affirmative action: Sabotage in handicap contests. Journal of Economic Behavior and Organization, 133, 273-284.

Chen, K. P. (2003). Sabotage in promotion tournaments. Journal of Law, Economics and Organization, 19, $119-140$.

Chowdhury, S. M., \& Gürtler, O. (2015). Sabotage in contests: A survey. Public Choice, 164, 135-155.

Deutscher, C., Frick, B., Gürtler, O., \& Prinz, J. (2013). Sabotage in tournaments with heterogeneous contestants: Empirical evidence from the soccer pitch. Scandinavian Journal of Economics, 115, $1138-1157$.

Dye, R. A. (1984). The trouble with tournaments. Economic Inquiry, 22, 147-149.

Esteban, J., \& Ray, D. (2001). Collective action and the group-size paradox. American Political Science Review, 95, 663-672.

Garicano, L., \& Palacios-Huerta, I. (2014). Making the beautiful game a bit less beautiful. Beautiful game theory-how soccer can help economics (pp. 124-150). Princeton: Princeton University Press.

Gershkov, A., Li, J., \& Schweinzer, P. (2009). Efficient tournaments within teams. The RAND Journal of Economics, 40, 103-119.

Gürtler, O. (2008). On sabotage in collective tournaments. Journal of Mathematical Economics, 44, 383-393.

Gürtler, O., \& Münster, J. (2010). Sabotage in dynamic tournaments. Journal of Mathematical Economics, $46,179-190$.

Harbring, C., \& Irlenbusch, B. (2005). Incentives in tournaments with endogenous prize selection. Journal of Institutional and Theoretical Economics, 161, 636-663.

Harbring, C., \& Irlenbusch, B. (2011). Sabotage in tournaments: Evidence from a laboratory experiment. Management Science, 57, 611-627.

Harbring, C., Irlenbusch, B., Kräkel, M., \& Selten, R. (2007). Sabotage in corporate contests-an experimental analysis. International Journal of the Economics of Business, 14, 367-392.

Holmström, B. (1982). Moral hazard in teams. The Bell Journal of Economics, 13, 324-340.

Itoh, H. (1991). Incentives to help in multi-agent situations. Econometrica, 59, 611-636.

Konrad, K. A. (2000). Sabotage in rent-seeking contests. Journal of Law, Economics and Organization, 16, $155-165$.

\footnotetext{
${ }^{16} \mathrm{We}$ are referring to the football game or war interpretations here. If we consider the election interpretation, we would expect that $\mu_{1}^{a a}<\mu_{1}^{d a}$, since player $a$ in team 1 is now closer to player $a$ in team 2 than player $d$ in team 1 is.
} 
Lazear, E. P. (1989). Pay equality and industrial politics. Journal of Political Economy, 97, 561-580.

McAfee, R. P., \& McMillan, J. (1991). Optimal contracts for teams. International Economic Review, 32, 561-577.

Münster, J. (2007). Selection tournaments, sabotage, and participation. Journal of Economics and Management Strategy, 16, 943-970.

Nitzan, S. (1991). Collective rent dissipation. The Economic Journal, 101, 1522-1534.

Olson, M. (1965). The logic of collective action: Public goods and the theory of groups. Cambridge: Harvard University Press.

Vandegrift, D., \& Yavas, A. (2010). An experimental test of sabotage in tournaments. Journal of Institutional and Theoretical Economics, 166, 259-285.

Vander Veen, T. D. (1995). Optimal contracts for teams: A note on the results of McAfee and McMillan. International Economic Review, 36, 1051-1056.

Publisher's Note Springer Nature remains neutral with regard to jurisdictional claims in published maps and institutional affiliations. 